\title{
Processi per il controllo delle emissioni industriali: verso i metodi biologici
}

\author{
Carmen Granato ${ }^{1}$ \\ ${ }^{1}$ Tecnologie per l'ambiente
}

\begin{abstract}
Come risultato della crescente consapevolezza sociale e sviluppo della legislazione, affrontare il problema legato al fastidio degli odori dovuti alle emissioni di composti organici volatili (COV) provenienti da una vasta gamma di impianti industriali, diventa spesso obbligatorio. Per il controllo delle suddette emissioni e per la rimozione, sono disponibili diverse soluzioni che si basano su processi chimico/fisici (assorbimento o lavaggio) che hanno bisogno a loro volta di ulteriori trattamenti oppure su processi biologici e processi avanzati di ossidazione $(\mathrm{AOP})$ che presentano soluzioni più efficaci. Nella prima parte dello studio sono stati confrontati tra loro i principali processi per il trattamento delle emissioni odorigene, attraverso un modello di confronto a coppie prendendo in considerazione efficacia, prestazioni di processo, possibilità di recupero e impatti ambientali - Negli ultimi decenni si è assistito a un significativo aumento dell'interesse per i processi di trattamenti biologici quali biofiltri e biotrickling filter in quanto più sostenibili dei convenzionali processi chimico/fisici. Questi ultimi, sono stati anch'essi esaminati attraverso un modello di confronto a coppie, associando, inoltre, un approccio per la selezione del processo in maniera preventiva, per mezzo di un algoritmo semplice basato su un modello ad albero decisionale. I risultati rilevano che la filtrazione biotrickling è il metodo più efficace per la rimozione dei COV idrofili, mentre i biofiltri consentono una rimozione più efficace per i COV idrofobici . Questo articolo può essere utile quando si seleziona, in maniera preliminare e basandosi solo su dati input, una procedura di trattamento per i composti odorosi rispetto ad un'altra.
\end{abstract}

\section{II problema delle emissioni degli impianti indus- triali e principali processi per il trattamento}

Con la popolazione e livelli di industrializzazione sempre più crescenti, la domanda per le tecnologie sostenibili di controllo degli odori e delle emissioni di impianti industriali (Figura 1) diventa sempre più importante, al fine di garantire maggior aria pulita e minori fastidi intorno alle fonti di emissione. $^{1}$ 


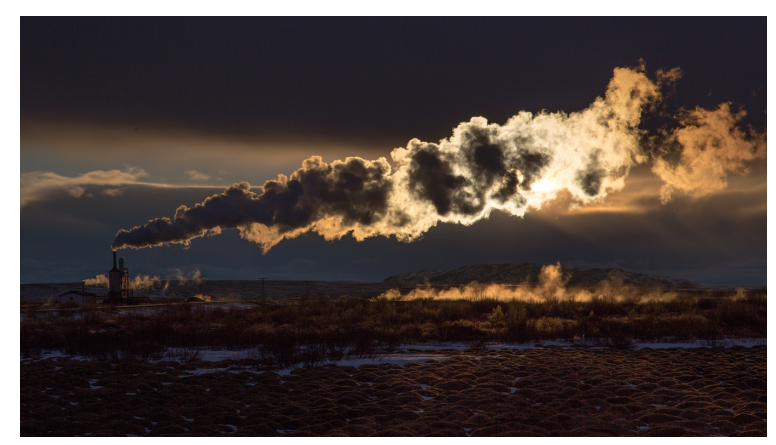

Figure 1: Emissioni di impianti industriali

Fonte di emissione degli impianti, sono i composti organici volatili (COV ), inclusi tra i gas organici prioritari composti da BTEX (benzene, toluene, etilbenzene e xilene) che classifica 78 su 275 sostanze identificate come le più pericolose per salute umana. ${ }^{2}$

Un' esposizione a lungo termine può far apparire gravi danni alla salute umana come leucemia e problemi respiratori, possono inoltre causare riscaldamento globale. ${ }^{3}$ Questo particolare impatto può portare a una scarsa qualità di vita e generare una percezione di un rischio per la comunità. Le emissioni di odori sono considerate inquinanti atmosferici e per questo motivo, tutto ciò ha richiesto attenzione immediata.

Le norme nazionali sulle emissioni di odori (D.Lgs 152/2006) sono state aggiornate di recente, richiedendo norme più rigorose (D. Lgs. 183/2017) rispetto a prima. Numerosi metodi di trattamento, comprese le tecnologie fisiche, chimiche e biologiche, sono stati sviluppati per limitare le emissioni di gas odorosi. ${ }^{6}$ Infatti i trattamenti di cui oggi si dispone sono vari:

- metodi fisici/chimici classici (come adsorbimento, assorbimento, condensazione, incenerimento o combustione catalitica): sono costosi e spesso generano inquinamenti secondari. ${ }^{7}$ Tuttavia trattano efficacemente i gas odorosi e la loro applicazione è idonea quando si ha a che fare con concentrazioni elevate. ${ }^{6}$ Sono molto utilizzati tra le varie tecnologie, essendo molto conosciuti;

- processi biologici e di ossidazione: sono in grado di promuovere la degradazione parziale o completa dei composti organici. Di conseguenza, questi processi non richiedono un ulteriore trattamento delle fasi. 8 Inoltre, stanno diventando sempre più popolari poiché consentono di purificare grandi flussi d'aria; ${ }^{7}$

- processi avanzati di ossidazione favoriscono la degradazione di un'ampia varietà di COV anche in presenza di elevati carichi in entrata; in ogni caso, il rilascio di sottoprodotti tossici e l'elevato consumo di energia rappresentano i principali svantaggi di questo tipo di processi. 98 
Dato che non esiste un metodo universale di purificazione dell'aria a causa della complessa composizione chimica dei COV e delle diverse condizioni di processo ${ }^{7}$, andando a confrontare i trattamenti attraverso un modello di confronto a coppie ${ }^{10}$, si è visto che ognuno presenta punti di forza e punti di debolezza; di conseguenza si vuole cercare di definire una metodologia per affrontare la scelta di una più adeguata tecnologia di trattamento per i gas di scarico provenienti da fonti industriali ${ }^{8}$, ponendo una maggiore attenzione verso i metodi biologici.

\section{Valutazione dei metodi per il controllo delle emissioni industriali}

Utilizzando un confronto a coppie tra quattro criteri diversi (efficacia, prestazioni di processo, possibilità di recupero e impatti ambientali) recuperati dalla letteratura scientifica, il metodo si basa su tre passaggi. Nel primo step si delineano i criteri gerarchicamente, con il secondo step si assegna un peso a questi ultimi mediante un confronto a coppie e nel terzo step si assegna un punteggio alle varie alternative attraverso la seguente equazione:

$$
\begin{aligned}
& S\left(A_{k}\right)= \\
& \sum_{S_{i}}^{n} \omega_{i} .
\end{aligned}
$$

funzione del peso del criterio $\omega_{i}$ e del punteggio dell'alternativa $A_{k}$ rispetto al criterio $\left(\mathrm{S}_{i}\right)$.

Ai criteri di efficacia e di impatto ambientale sono stati assegnati i pesi più alti e sono state evidenziate le debolezze e i punti di forza dei processi studiati. Per ciascun criterio, l'indicatore corrispondente è stato convertito come indice, in seguito alla procedura di normalizzazione. Tutti gli indicatori sono stati normalizzati secondo il metodo "high is better". 8 I risultati indicano che i processi di ossidazione sono caratterizzati dalla massima efficacia ma con impatti ambientali maggiori mentre la biofiltrazione ha permesso di ottenere un'alta efficienza anche se quest'ultima dipende dalle concentrazioni di ingresso dei COV. Tutte le soluzioni studiate, specificate in Figura 2, hanno rivelato risultati comparabili:

quando sono previste concentrazioni basse in ingresso, la biofiltrazione potrebbe essere considerata la migliore opzione in quanto questo tipo di processo è stato in grado di raggiungere un'alta efficienza con bassi impatti ambientali ed economici ${ }^{11} 8$. Al contrario, quando sono richieste alte prestazioni, le soluzioni più efficaci sono il plasma, la foto-catalisi e l'ossidazione UV. La maggior parte degli AOP presenta importanti limiti 


\begin{tabular}{|c|c|c|c|c|c|}
\hline Processi & \multicolumn{4}{|c|}{ Criteri } & TOT \\
\hline & 1 & 2 & 3 & 4 & \\
\hline Assorbimento & 0,68 & 0,04 & 0,67 & 0,75 & 0,58 \\
\hline Adsorbimento & 0,46 & 0,19 & 0,67 & 0,75 & 0,53 \\
\hline Biofiltrazione & 0,44 & 0,62 & 0,00 & 0,75 & 0,54 \\
\hline Condensazione & 0,60 & 0,54 & 0,67 & 0,50 & 0,56 \\
\hline Ossidazione Catalitica & 0,80 & 0,84 & 0,33 & 0,25 & 0,57 \\
\hline Ossidazione Termica & 0,61 & 0,50 & 0,33 & 0,25 & 0,58 \\
\hline Plasma & 0,96 & 0,77 & 0,00 & 0,25 & 0,58 \\
\hline Fotocatalisi & 0,96 & 0,52 & 0,00 & 0,25 & 0,53 \\
\hline Ossidazione UV dell'ozono & 0,96 & 0,77 & 0,00 & 0,25 & 0.58 \\
\hline
\end{tabular}

Figure 2: Punteggio delle possibili soluzioni di trattamento per ogni criterio (adattata da Oliva et all 2018)

se utilizzati singolarmente a causa della loro cinetica piuttosto lenta. Per ovviare a questa carenza, l'applicazione degli AOP viene effettuata contemporaneamente per beneficiare di possibili effetti sinergici.

Per ridurre le emissioni di sottoprodotti si potrebbe implementare un processo combinato, ovvero i processi avanzati di ossidazione (AOP) fungono da pre-trattamento ai processi biologici, andando a migliorare la biotrattabilità dei composti organici volatili (COV) e controllare l'accumulo di biomassa. In base a ciò, si vuole effettuare una valutazione mirata ai metodi biologici che comprendono biofiltri convenzionali (BF), filtri biotrickling (BTF) e bioscrubber (BS), valutandoli e utilizzando ancora una volta il confronto a coppie seguito da una procedura ad albero decisionale. Segue una breve descrizione dei metodi biologici.

La biofiltrazione è una tecnologia in cui i gas di scarico, contenenti composti organici volatili biodegradabili (COV) o composti atmosferici inorganici e tossici, sono trattati attraverso un materiale biologicamente attivo. ${ }^{13}$

A seconda del metodo di gestione del processo biologico per il trattamento del gas, i microrganismi possono essere sospesi nel sorbente (bioscrubbers) o posizionati sulla superficie di un materiale solido (biofiltri). ${ }^{14}$

Poichè i biofiltri forniscono uno scarso controllo dei nutrienti e del pH, gli ingegneri li hanno modificati con l'aggiunta di un bioreattore a riempimento di liquido per fornire un sistema chiamato filtro biotrickling (BTF). ${ }^{15}$

Per caratterizzare i metodi biologici si è agito come prima ovvero andando a selezionare quattro criteri: efficienza / prestazioni del processo, costi, aspetti tecnici e impatto ambientale. Ciascuno dei criteri selezionati è stato diviso in criteri di secondo ordine come si riporta in Figura 3:

Agendo esattamente come nel caso precedentemente analizzato, per valutare i processi biologici, bisogna introdurre anche le differenze nel carat- 


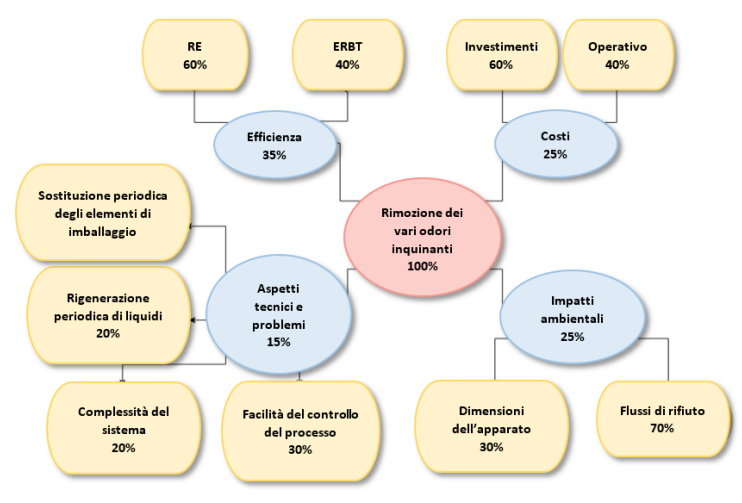

Figure 3: Gerarchia di criteri e sotto criteri selezionati (adattata da Gospodarek et all 2019)

tere idrofilo dei composti che sono state stimate usando la costante della legge di Henry. Maggiore è la costante della legge di Henry, maggiore è la volatilità e minore è la solubilità di un composto.

I risultati riepilogativi per ciascun metodo di trattamento studiato indicano che: i biofiltri convenzionali e i filtri per biotrickling mostrano prestazioni simili e buone per il trattamento dei composti idrofobici. I filtri biotrickling sono superiori in termini di rimozione dei composti idrofili, mentre $i$ bioscrubbers presentano prestazioni moderate o basse rispetto a BF e BTF.

Sulla base di dati di letteratura, è stato introdotto un albero decisionale per la scelta del metodo biologico migliore, ovvero un algoritmo non arbitrario per la selezione preliminare di un metodo di deodorizzazione dell'aria, ed è presentato nella Figura 4. L'albero decisionale presentato mostra, in base ai dati di input utilizzati, che solo due parametri sono importanti nella scelta del metodo corretto, ossia la concentrazione in ingresso di un composto e l'idrofobicità del composto, rappresentata dalla costante di legge di Henry. Nello sviluppo del modello di scelta finale, sono state incluse solo le prestazioni del processo, mentre è stata esclusa l'analisi dei costi. 10

L'output dell'albero decisionale suggerisce l'utilizzo di biofiltri convenzionali per il trattamento di flussi altamente concentrati (concentrazione superiore a $2500 \mathrm{mg}[?] \mathrm{m}^{-3}$ ). Per i flussi con concentrazioni di composti odorosi inferiori a $2500 \mathrm{mg}[?] \mathrm{m}^{-3}$, la filtrazione con biotrickling e un metodo piu adatto rispetto agli altri due. I bioscrubbers possono essere usati per composti caratterizzati dalla costante della legge di Henry tra 0,0075 e 0,62 $\mathrm{mol}[?] \mathrm{m}^{-3}[?] \mathrm{Pa}^{-1}$ e per concentrazioni di ingresso inferiori a $2500 \mathrm{mg}^{*} \mathrm{~m}^{-3}$. 


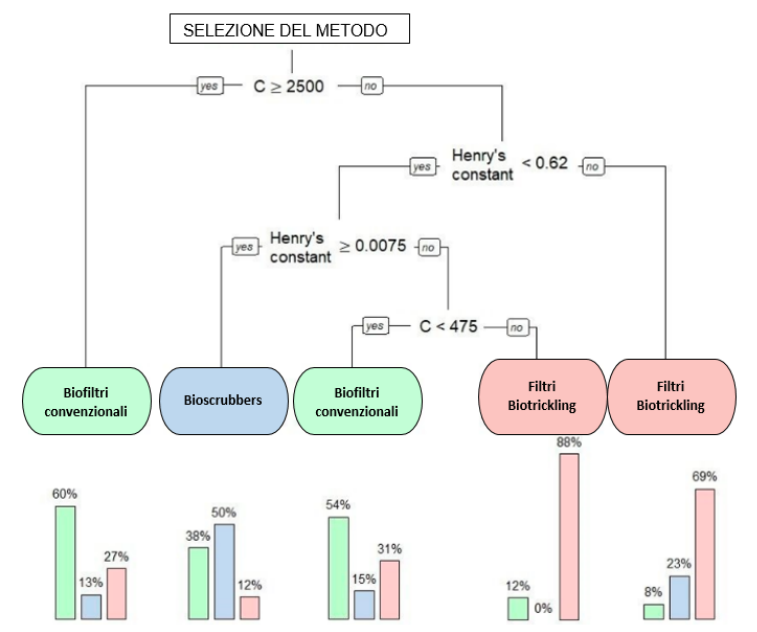

Figure 4: Albero decisionale sviluppato per la selezione del metodo di trattamento biologico più adatto $\left(\mathrm{C}=\right.$ concentrazione dell'iniettore $\mathrm{mg}[?] \mathrm{m}^{-3}$; costante di Henry, mol[?] $\mathrm{m}^{-3}[?] \mathrm{Pa}^{-1}$ ) (adattata da Gospodarek et all 2019 )

\section{Conclusioni}

Attraverso questo studio, si e voluto presentare un prototipo di un modello decisionale preventivo ai fini del trattamento dei composti odorosi. In particolare partendo da alcuni dati ricavati dalla letteratura, si e prima fatto un confronto a coppie per confrontare i metodi biologici quali biofiltri convenzionali biotrickling e bioscrubbers. Da questi dati di letteratura si e passati ad un algoritmo di decisione di tipo ad albero il quale ci ha fornito un metodo per scegliere il tipo di trattamento da effettuare, per composti odorosi idrofili e idrofobici che si basa soprattutto sulla concentrazione iniziale e sul parametro di Henry. Questo fornisce una base per un prossimo modello basato sull'estensione dei dati di input, il quale consentira una rapida selezione del metodo appropriato di purificazione dell'aria inquinata per composti specifici.

In un modello futuro, si terra conto anche delle dimensioni dell'apparato, della geometria, delle specie microbiche e di tutti i parametri di processo importanti con la prospettiva di facilitare la selezione del metodo di trattamento. ${ }^{10}$ 


\section{References}

1.Vikrant, K. et al.. Bio-filters for the Treatment of VOCs and Odors: A Review. Asian Journal of Atmospheric Environment 11, 139-152 (2017).

2.Oliva, G. et al.. Comparative evaluation of a biotrickling filter and a tubular photobioreactor for the continuous abatement of toluene. Journal of Hazardous Materials 380, 120860 (2019).

3.Gopal, N. R. \& Satyanarayana, S. V. Cost analysis for removal of VOCs from water by pervaporation using NSGA-II. Desalination 274, 212-219 (2011).

4.Gostelow, P., Parsons, S. A. \& Stuetz, R. M. Odour measurements for sewage treatment works. Water Research 35, 579-597 (2001).

5.Zarra, T., Galang, M. G., Ballesteros, F., Belgiorno, V. \& Naddeo, V. Environmental odour management by artificial neural network - A review. Environment International 133, 105189 (2019).

6.Li, W. et al.. Variations in microbial community structure and functional gene expression in bio-treatment processes with odorous pollutants. Scientific Reports 9, (2019).

7.Gaszczak, A., Bartelmus, G., Burghardt, A., Rotkegel, A. \& Sarzynski, R. Experiments and modelling of a biotrickling filter (BTF) for removal of styrene from airstreams. Journal of Chemical Technology \& Biotechnology 93, 2659-2670 (2018).

8.Oliva, G. et al.. Comparative Analysis of AOPs and Biological Processes for the Control of VOCs Industrial Emissions. Chemical engineering transaction 68, (2018).

9.Akmirza, I. et al.. Anoxic biodegradation of BTEX in a biotrickling filter. Science of The Total Environment 587-588, 457-465 (2017).

10.Gospodarek, M., Rybarczyk, P., Szulczynski, B. \& Gebicki, J. Comparative Evaluation of Selected Biological Methods for the Removal of Hydrophilic and Hydrophobic Odorous VOCs from Air. Processes 7, 187 (2019).

11.Munoz, R., Malhautier, L., Fanlo, J.-L. \& Quijano, G. Biological technologies for the treatment of atmospheric pollutants. International Journal of Environmental Analytical Chemistry 95, 950-967 (2015).

12.Sharfalddin, A., Alzahrani, E. \& Alamoudi, M. Investigation of the synergism of hybrid advanced oxidation processes with an oxidation agent to degrade three dyes. Research on Chemical Intermediates 43, 2587-2601 (2016).

13.Leson, G. \& Winer, A. M. Biofiltration: An Innovative Air Pollution 
Control Technology For VOC Emissions. Journal of the Air \& Waste Management Association 41, 1045-1054 (1991).

14.Wysocka, I., Gebicki, J. \& Namiesnik, J. Technologies for deodorization of malodorous gases. Environmental Science and Pollution Research 26, 9409-9434 (2019).

15. Koutinas, M., Peeva, L. G. \& Livingston, A. G. An attempt to compare the performance of bioscrubbers and biotrickling filters for degradation of ethyl acetate in gas streams. Journal of Chemical Technology \& Biotechnology 80, 1252-1260 (2005). 